\title{
Photorefractive light needles in glassy nanodisordered KNTN
}

\author{
D. Pierangeli, ${ }^{1}$ J. Parravicini, ${ }^{1,2}$ F. Di Mei, ${ }^{1,3}$ G. B. Parravicini, ${ }^{2,4}$ A. J. Agranat, ${ }^{5}$ and E. DelRe ${ }^{1,2, *}$ \\ ${ }^{1}$ Physics Department, University of Rome Sapienza, 00185 Rome, Italy \\ ${ }^{2} I P C F-C N R$, University of Rome Sapienza, 00185 Rome, Italy \\ ${ }^{3}$ Center for Life Nano Science at Sapienza, Istituto Italiano di Tecnologia, 00161 Rome, Italy \\ ${ }^{4}$ Physics Department, University of Pavia, 27100 Pavia, Italy \\ ${ }^{5}$ Applied Physics Department, Hebrew University of Jerusalem, 91904, Israel \\ ${ }^{*}$ Corresponding author: eugenio.delre@uniroma1.it
}

Received October 17, 2013; revised February 10, 2014; accepted February 11, 2014;

posted February 11, 2014 (Doc. ID 199509); published March 14, 2014

\begin{abstract}
We study the formation of 2D self-trapped beams in nanodisordered potassium-sodium-tantalate-niobate (KNTN) cooled below the dynamic glass transition. Supercooling is shown to accelerate the photorefractive response and enhance steady-state anisotropy. Effects in the excited state are attributed to the anomalous slim-loop polarization curve typical of relaxors dominated by non-interacting polar-nano-regions. (c) 2014 Optical Society of America OCIS codes: (190.4720) Optical nonlinearities of condensed matter; (190.5330) Photorefractive optics; (190.6135) Spatial solitons.

http://dx.doi.org/10.1364/OL.39.001657
\end{abstract}

Nonlinear optics is generally performed in systems that start from equilibrium, where long-range order and coherence form the basis for strong response. Compositional disorder in a perovskite ferroelectric can turn it into a relaxor, a crystal where long-range order is frustrated but short-range order, in the form of polar-nanoregions (PNRs), is anomalously enhanced [1,2]. Relaxors appear to manifest a non-ergodic phase with dipolar-glass properties, with a dielectric response that depends on previous history [3]. In many respects, the perfect lattice can be considered to host an embedded glass-formingliquid, i.e., with relaxational properties that are described by the Adam-Gibbs model [4]. Apart from fundamental challenges $[\underline{5}, 6]$, relaxors offer the promise of applications in a variety of fields, such as tunable capacitors and memory chips.

In terms of optics, one basic question is what happens to linear and nonlinear optical propagation inside a relaxor. Recent experiments in relaxors are focusing on transparent samples with high optical quality [7]. This presents the unique possibility of tapping into the complex-solid behavior to affect light control [,,], design novel optical devices [10], and, in general, uncover new wave phenomena, such as the recently demonstrated diffraction cancellation associated to scale-free optics in supercooled potassium-lithium-tantalate-niobate (KLTN) [11-14].

Photorefractive ferroelectrics at equilibrium support 2D optical spatial solitons, beams for which diffraction is compensated by self-focusing $[15,16]$. These waves not only represent a rare example of a higher-than-onedimensional solitary wave [17], but, amounting to fiberlike guiding channels that are self-written into the bulk of the crystal, support miniaturized optical switching and routing circuits $[18,19]$. Potential applications range from massive switching matrices in communication networks [20] to complex polarization-sensitive circuitry for linear quantum computing simulation $[21,22]$.

In this Letter, we experimentally investigate the formation of $2 \mathrm{D}$ solitons in compositionally disordered potassium-sodium-tantalate-niobate (KNTN), a crystal in which we have recently observed soliton aging [23]. In our experiments, we show how changing the cooling rate, with which we reach the final operation temperature, we are able to fundamentally change the solitonsupporting nonlinearity, selectively shifting from a slow isotropic response to a fast anisotropic one. We are able to attribute this shift to the selective activation of the slim-loop polarization curve characteristic of quenched disordered dipole systems. Apart from the basic fact that we are thus able to make, on command, a spatial soliton be anisotropic and hence, for example, change its polarization sensitivity, our scheme demonstrates a means to tune optical nonlinearity at levels that were previously believed to require the design of new crystals. More fundamentally, our findings demonstrate how glassy response can change not only the crystal-dependent parameters mediating the light-matter interaction, but also the nature of the nonlinearity.

We have grown our sample of $\mathrm{K}_{1-x} \mathrm{Na}_{x} \mathrm{Ta}_{1-y} \mathrm{Nb}_{y} \mathrm{O}_{3}$ through the top-seeded solution method and extracted a zero-cut $1.17^{(x)} \times 1.90^{(y)} \times 2.43^{(z)} \mathrm{mm}$ specimen with $\mathrm{K}_{0.89} \mathrm{Na}_{0.11} \mathrm{Ta}_{0.63} \mathrm{Nb}_{0.37}$. The KNTN is also Cu-doped (0.001 atoms per mole) and manifests strong photorefractive response for visible wavelengths. In order to identify the temperature range of existence of permanent dynamic PNRs in the relaxor, we perform dielectric spectroscopy measurements using a standard LCR meter scheme for different frequencies and a thermal chamber. Measurements are in a quasi-static regime, i.e., the cooling/heating rate for these spectroscopy experiments is $\alpha \simeq \pm 1 \mathrm{mK} / \mathrm{s}$. The results of the dielectric constant measurements are reported in Fig. 1. The temperature $T^{*}=305 \mathrm{~K}$, below which the mean-field Curie-Weiss law $\epsilon_{r}=C /\left(T-T_{C}\right)$ (dashed line) is violated, marks the PNRs onset as confirmed by dielectric dispersion in the real part of the permittivity below this value [inset of Fig. 1], i.e., where the relaxor behavior affects the dielectric response $[\underline{24}, \underline{25}]$ and, hence, photorefraction through the electro-optic effect. At $T_{\max }=285 \mathrm{~K}$, in turn, 


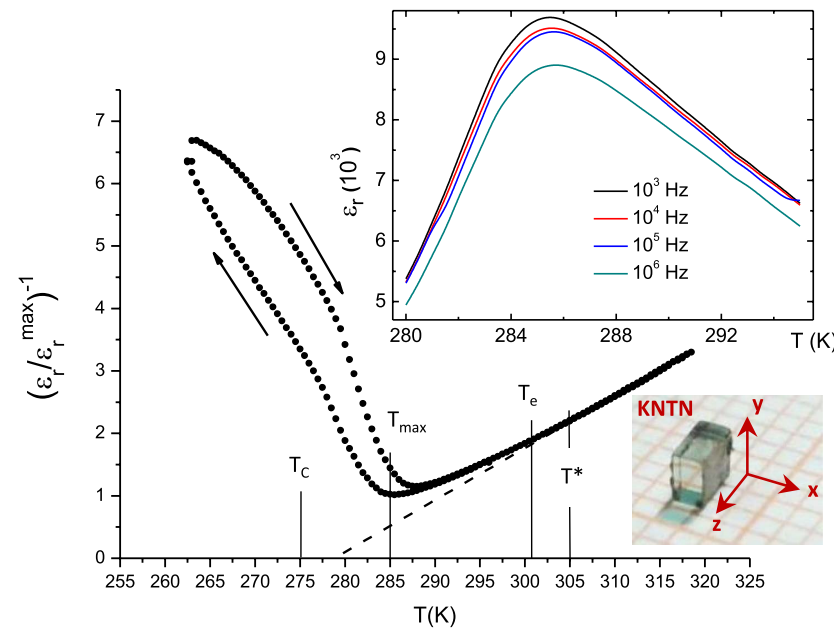

Fig. 1. Low-frequency $(1 \mathrm{KHz})$ measurement (circles) of the real part of the dielectric constant of the KNTN sample manifesting marked thermal hysteresis (arrows indicate cooling/ heating curves) below $T^{*}$ and a breaking of Curie-Weiss mean-field behavior (dashed line) in the region $T_{\max }<T<T^{*}$ $\left(\epsilon_{r}^{\max }=9.5 \cdot 10^{3}\right.$, decreasing temperature loop). In the insets, the detected dispersion in $\epsilon_{r}$ near $T_{\max }$ and a photograph of the specific KNTN sample.

large scale micrometer-sized ferroelectric clusters form that inhibit optical propagation, whereas $T_{C}=275 \mathrm{~K}$. Optically accessible glassy physics as a consequence of the cooling protocols (described below) is detected in the region $T_{\max }<T<T^{*}$.

In the optical scheme, the crystal is in thermal contact with a Peltier junction, which allows cooling protocols at various constant cooling rates, from a minimum of $\alpha_{\min }=$ $0.0025 \mathrm{~K} / \mathrm{s}$ to the maximum value of $\alpha_{\max }=0.07 \mathrm{~K} / \mathrm{s}$. In each experiment, we heat the sample at the "warm" $T_{i}=$ $310 \mathrm{~K}$ for $30 \mathrm{~min}$ and then enact a cooling at a fixed rate $\alpha$ to the final operation temperature $T_{e}=301 \mathrm{~K}$. An $x$ polarized (with reference to the crystal axes, see Fig. 1) $2 \mathrm{~mW}$ Gaussian beam from a doubled Nd:YAG laser $(\lambda=532 \mathrm{~nm})$ is expanded to approximately $10 \mathrm{~mm}$ diameter and divided by a 50/50 beam splitter. The transmitted beam is attenuated through a tunable neutral-density filter and focused down by an $f=150 \mathrm{~mm}$ spherical lens. The polarization of the reflected beam is rotated, using a $\lambda / 2$ waveplate, from the $x$ to the vertical $y$ plane. A second beam splitter recombines the two beams along the $z$ axis. The converging beam is focused onto the input facet of the crystal and diffracts as it propagates to the output facet. A lens after the crystal projects selectively the input and output facet onto a CCD. On the two lateral $x$ facets electrodes are applied that deliver a bias voltage $V$, giving rise to a bias electric field in the sample of $E=V / L_{x}$ (with $L_{x}=1.17 \mathrm{~mm}$ ).

In Fig. 2, we report results of $2 \mathrm{D}$ beam self-trapping, both for slow cooling to $T_{e}$, with $\alpha=\alpha_{\min }$, and rapid cooling, with $\alpha=\alpha_{\max }$. As shown in Fig. 2(a), the input round $\Delta x_{\text {in }}=\Delta y_{\text {in }}=8 \mu \mathrm{m}$ FWHM beam diffracts at the output facet to a $\Delta x=27 \mu \mathrm{m} \Delta y=21 \mu \mathrm{m}$ pattern. The distorted speckle-like distribution in the $V=0$ output is caused by inhomogeneities in the linear response (as established by simply translating the sample laterally, in which case also the output ellipticity can be readily inverted). For a ratio between the peak input beam intensity $I_{p}$ and average
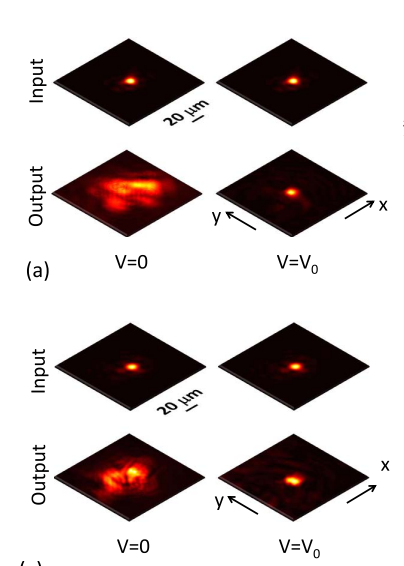

(c)
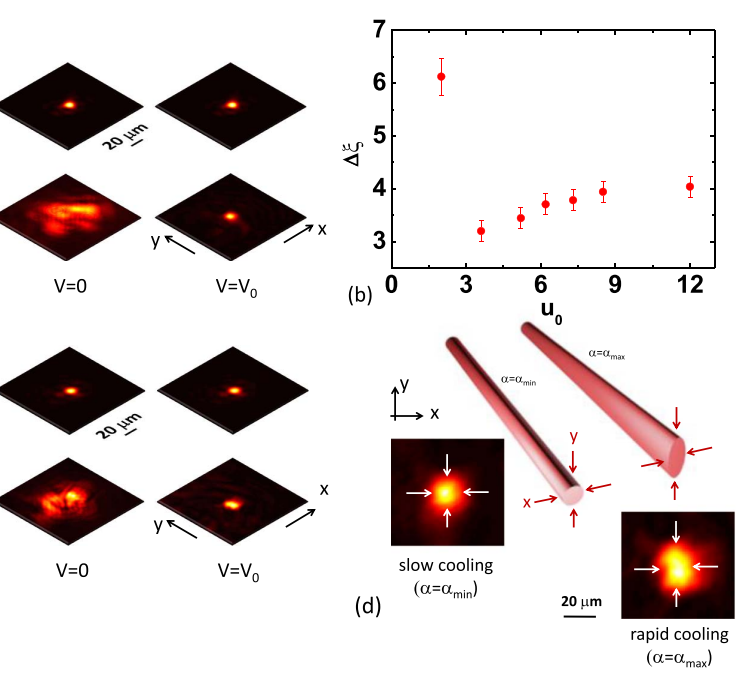

Fig. 2. Round versus elliptical 2D needles in KNTN. Slow cooling: (a) input and output intensity distributions at steady-state without and with applied bias voltage. (b) Existence conditions for $\Delta x=\Delta y \simeq \Delta x_{\mathrm{in}}$, in the soliton normalized amplitude-width parameter space (see text). Rapid cooling: (c) the input round $\Delta x_{\text {in }}=\Delta y_{\text {in }}=8 \mu \mathrm{m}$ beam diffracts to and average FWHM of $\Delta y=25 \mu \mathrm{m}$ for $V=0$ and converges to an elliptical beam profile for $V=V_{0}$, where only the $x$ direction traps to the initial launch size. No values of $V$ lead to a round soliton of the same size as the input. (d) Comparison of slow and fast cooling output intensity distributions at steady-state (top) and representation of inferred beam evolution (bottom).

intensity of the copropagating $y$ polarized (background) beam $I_{b}$ of $I_{p} / I_{b} \equiv u_{0}^{2}=29$ and an applied voltage $V_{0}=475 \mathrm{~V}$, the beam forms a stable nonspreading beam of the same input FWHM, a phenomenon that greatly retraces what is known for $2 \mathrm{D}$ screening solitons [26]. Changing launch $u_{0}$ and applied voltage $V_{0}$, imposing that a round $\Delta x=\Delta y=\Delta x_{\text {in }}$ output intensity distribution form with the same input FWHM leads to the identification of a $2 \mathrm{D}$ soliton "existence curve" in the beam parameter space [27], as reported in Fig. 2(b). We introduce the normalized soliton width $\Delta \xi[28,29]$. Assuming a round soliton so that $\Delta x=\Delta y, \xi=x / d, d=1 / \sqrt{-2 k b}$, $b=\left(k / n_{0}\right) \Delta n_{0}, \quad n=n_{0}+\Delta n(I), \quad \Delta n(I)=-\Delta n_{0}(1+$ $\left.I / I_{b}\right)^{-2}, \quad$ and $\quad \Delta n_{0}=(1 / 2) n_{0}^{2} g_{\text {eff }} \epsilon_{0}^{2}\left(\epsilon_{r}-1\right)^{2} E_{0}^{2}$. Here $E_{0}=V_{0} / L_{x}, k=2 \pi n_{0} / \lambda$ is the optical wave number, $n_{0}=2.31$ is the unperturbed index of refraction, $I$ is the spatially resolved optical intensity distribution, $g_{\text {eff }}=$ $0.14 \mathrm{C}^{-2} \mathrm{~m}^{4}$ is the effective quadratic electro-optic coefficient, and $\epsilon_{r}\left(T_{e}\right)=4800$ is the quasi-static dielectric constant. Results reported in Fig. 2(b) agree with $2 \mathrm{D}$ screening soliton phenomena in centrosymmetric systems [29,30]. In Fig. 2(c), we report results for the rapid cooling case of $\alpha=\alpha_{\max }$. As shown in Fig. 2(c), in the same conditions of Fig. 2(a), the beam self traps at a steady state to a beam with an elliptical profile, with $\Delta x=\Delta x_{\text {in }}=8 \mu \mathrm{m}$ and $\Delta y=12 \mu \mathrm{m}$. Repeating the experiment for all accessible values of $V_{0}<1 \mathrm{KV}$ indicates that $\Delta x$ attains the same value as the input, forming a soliton, only for the $V_{0} \simeq 480 \mathrm{~V}$ (compatible with the slow cooling experiment), and in these conditions the beam is elliptical, as illustrated in Fig. 2(d). Repeating the experi-

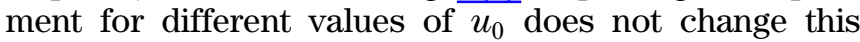
picture in the investigated range $2<u_{0}<12$. We observe 


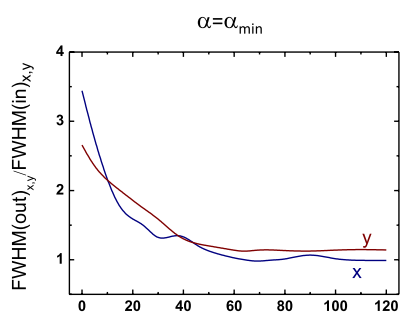

(a)

$\mathrm{t}(\min )$

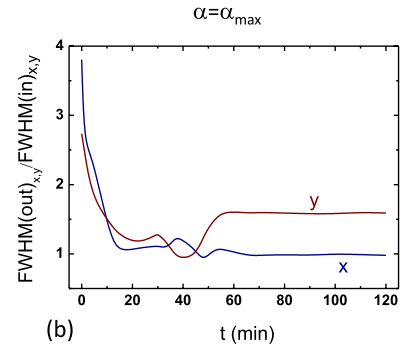

Fig. 3. Rapid cooling accelerates the photorefractive effect. (a) Time evolution of output beam FWHM normalized to input in the $x$ and $y$ directions in the slow-cooling case. (b) A more than four-fold speeding of self-trapping in the rapid-cooled sample.

a convergence of the beam to a self-trapped state in the $x$ direction, but not in the $y$, as illustrated in the bottom inset of Fig. 2(d).

We report in Fig. 3 the comparison between the slow and fast cooling experiments as these develop in time toward a steady state. Interestingly, the time scale of the two processes, measured taking the $90 \%$ and $10 \%$ values of $\Delta x-\Delta x_{\mathrm{in}}$, decreases from $\tau_{\alpha_{\min }} \simeq 48 \mathrm{~min}$ to $\tau_{\alpha_{\max }} \simeq 11 \mathrm{~min}$, a change that may be associated with a different conductivity in the two cases.

The annealing/quenching impacts on a debated and important aspect of photorefractive solitons, i.e., the role of anisotropy [31-33]. The zero-field-readout of the underlying pattern is reported in Figs. 4(a) and 4(b) [32,33]. Propagation properties are detected with $\bar{V}=0$ after the space charge has formed, before it can redistribute [34]. The patterns are what you observe after the soliton of Fig. 2 has formed but with no bias applied. Consistently with literature in conventional ferroelectrics, in the slow-cooling case that leads to round beams, the anisotropy is strongly influenced by nonlocality, as testified by the breaking of symmetry in the $x$ direction
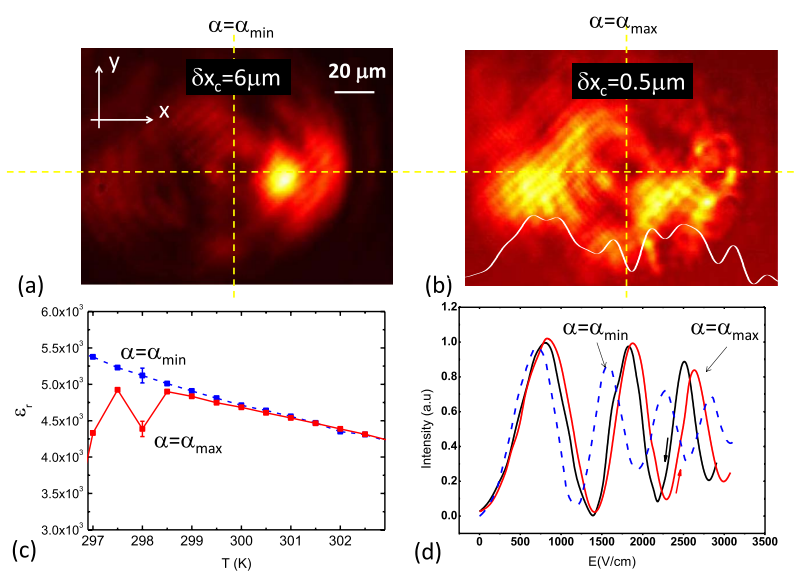

Fig. 4. Zero-field readout $(V=0)$ in the (a) slow and (b) rapid cooling cases. Defining $\bar{x} \equiv \int I(x, y) x \mathrm{~d} x \mathrm{~d} y / \int I(x, y) \mathrm{d} x \mathrm{~d} y$ the center of the pattern, $\delta x_{c}$ the shift of $\bar{x}$ for $V=V_{0}$ (crossing of dashed lines), and for $V=0, \delta x_{c}=6 \mu \mathrm{m}$ and $0.5 \mu \mathrm{m}$ in the two conditions, respectively. (c) Comparison of $\epsilon_{r}$ data. (d) Cross-polarizer transmission $I_{\text {out }} / I_{\text {in }}$ versus applied $E$. The slight shift in the pattern for increasing bias fields (red line) and for decreasing ones (black curve) quantifies a small hysteresis, whereas the decrease in fringe visibility is caused by depolarization [8]. of Fig. 4(a). Equally consistently, in the rapidly cooled case, where elliptical beams are observed, the symmetry is restored, meaning that the role of nonlocal components is attenuated [Fig. 4(b)]. Beam anisotropy is strongly influenced by the specific $P$ versus $E$ relationship, where $E$ and $P$ are the point-dependent electric field and macroscopic polarization, and this relation is known to change the cooling rate of the crystal $[10,11]$. The photorefractive model leads to the expression in [26]:

$$
\mathbf{E} \cdot\left(I_{b}+I\right) /\left(1+\nabla \cdot \mathbf{D} / q N_{a}\right)=g,
$$

where $\mathbf{D}=\epsilon_{0} \mathbf{E}+\mathbf{P}, g$ is a constant proportional to $E_{0}, q$ is the elementary charge of the photoexcited carriers, $N_{a}$ is the density of acceptor sites, charge diffusion is neglected, and the condition $N_{a} \ll N_{d}$ holds, where $N_{d}$ is the concentration of donor impurities. The larger the value of $\nabla \cdot \mathbf{D} / q N_{a}$, the more attenuated the anisotropy in the beam, the stronger the achievable soliton circularsymmetry [32]. Now $\nabla \cdot \mathbf{D}=\nabla \cdot\left(\epsilon_{0} \mathbf{E}+\mathbf{P}\right) \simeq \nabla \cdot \mathbf{P}$, a term that is fundamentally governed by the $\mathbf{P}$ versus $\mathbf{E}$ relation (in the simplified one-transverse-dimensional case, $\quad d P / d x=(d P / d E)(d E / d x))$. Even though $\epsilon_{r}$ (1 $\mathrm{KHz} 100 \mathrm{mV}$ signal) for the slow and rapid cooling cases leads to no appreciable difference at the operation temperature $T_{e}$ [Fig. 4(c)], a more detailed picture emerges from cross-polarizer experiments [Fig. 4(d)]. The sample is placed between two polarizers with mutually orthogonal axes, at $45 \mathrm{deg}$ with respect to the crystal principal axes (the crystal is zero-cut). The transmission of light is, hence, possible because of the electro-optic response to the $x$ directed bias field according to the law $I_{\text {out }} / I_{\text {in }}=\sin ^{2}(\Delta \phi / 2), \Delta \phi=\Delta n(2 \pi / \lambda) L_{z}$. Measuring transmission leads to the value of $\Delta \phi(E)$ and to the $P$ versus $E$ relation through $\Delta n=-(1 / 2) n_{0}^{2} g_{\text {eff }} P^{2}$. As reported in Fig. 4(d), where the residual birefringence at zero field has been compensated, for the typical values of space-charge field involved in our experiments $\left(E \sim 4 \cdot 10^{5} \mathrm{~V} / \mathrm{m}\right)$, the response decreases and saturates in the supercooled case (full lines) compared to the slowcooling case (dashed line), i.e., the fringe pattern in the quenched case lags ever more behind the annealed fringe pattern. This sublinear $P$ versus $E$ relation is typical of relaxors and their "slim loop" hysteresis curve above $T_{\max }[1,2]$. This can filter out the spatial dependence of $P$ caused by the spatial dependence of the solitonsupporting light induced $E$, and reduce the $\nabla \cdot \mathbf{D} / q N_{a}$ term. As reported in Fig. 4(d), we also detect a decrease in fringe visibility $\left(I_{\max }-\overline{I_{\min }} /\left(I_{\max }+I_{\min }\right)\right)$ for decreasing cooling rates indicating, in agreement with previous literature [ $\underline{8}, \underline{9}]$, that underlying PNRs grow in size, reaching the wavelength scale unless rapid cooling is used. Operating closer to the $T_{\max }$ or applying alternating bias fields during super-cooling can also fundamentally change the $P$ versus $E$ relation causing, for example, a giant electro-optic effect [9]. Evidently, a full theoretical model of photorefraction and solitons in quenched relaxors requires further development.

In summary, we have detected and analyzed 2D spatial soliton beams in relaxor KNTN. Our findings side with the ever-growing evidence of how disorder, even in apparently negligible quantities, can dramatical change the macroscopic response of a crystal, as is known to 
occur in colossal magnetoresistance, piezoresistance, and electroresistance in perovskites [35].

Funding from grants PHOCOS-RBFR08E7VA, PRIN 2012BFNWZ2, and the "SUPERCONTINUUM" Sapienza project are acknowledged. A. J. A. acknowledges the support of the Peter Brojde Center for Innovative Engineering.

\section{References}

1. G. A. Samara, J. Phys. Condens. Matter 15, R367 (2003).

2. A. A. Bokov and Z.-G. Ye, J. Mater. Sci. 41, 31 (2006).

3. G. Burns and F. H. Dacol, Phys. Rev. B 28, 2527 (1983).

4. P. B. Ishai, C. E. M. de Oliveira, Y. Ryabov, Y. Feldman, and A. J. Agranat, Phys. Rev. B 70, 132104 (2004).

5. E. Donth, The Glass Transition (Springer-Verlag, 2001).

6. L. Leuzzi and T. M. Nieuwenhuizen, Thermodynamics of the Glassy State (Taylor \& Francis, 2008).

7. W. Ruan, G. Li, J. Zeng, L. S. Kamzina, H. Zeng, K. Zhao, L. Zheng, and A. Ding, J. Appl. Phys. 110, 074109 (2011).

8. A. Gumennik, Y. Kurzweil-Segev, and A. J. Agranat, Opt. Mater. Express 1, 332 (2011).

9. Y. Chang, C. Wang, S. Yin, R. C. Hoffman, and A. G. Mott, Opt. Lett. 38, 4574 (2013).

10. Y. Chang, C. Wang, S. Yin, R. C. Hoffman, and A. G. Mott, Opt. Express 21, 17760 (2013).

11. E. DelRe, E. Spinozzi, A. J. Agranat, and C. Conti, Nat. Photonics 5, 39 (2011).

12. J. Parravicini, F. Di Mei, C. Conti, A. J. Agranat, and E. DelRe, Opt. Express 19, 24109 (2011).

13. J. Parravicini, C. Conti, A. J. Agranat, and E. DelRe, Opt. Express 20, 27382 (2012).

14. J. Parravicini, C. Conti, A. J. Agranat, and E. DelRe, Opt. Lett. 37, 2355 (2012).

15. M. F. Shih, M. Segev, G. C. Valley, G. Salamo, B. Crosignani, and P. Di Porto, Electron. Lett. 31, 826 (1995).

16. M. F. Shih, P. Leach, M. Segev, M. H. Garrett, G. Salamo, and G. C. Valley, Opt. Lett. 21, 324 (1996).
17. Z. Chen, M. Segev, and D. Christodoulides, Rep. Prog. Phys. 75, 086401 (2012).

18. M. Morin, G. Duree, G. Salamo, and M. Segev, Opt. Lett. 20, 2066 (1995).

19. M. Asaro, M. Sheldon, Z. G. Chen, O. Ostroverkhova, and W. E. Moerner, Opt. Lett. 30, 519 (2005).

20. A. D'Ercole, E. Palange, E. DelRe, A. Ciattoni, B. Crosignani, and A. J. Agranat, Appl. Phys. Lett. 85, 2679 (2004).

21. A. D'Ercole, A. Pierangelo, E. Palange, A. Ciattoni, A. J. Agranat, and E. DelRe, Opt. Express 16, 12002 (2008).

22. L. Al Fares, F. Devaux, B. Guichardaz, and M. Chauvet, Appl. Phys. Lett. 103, 041111 (2013).

23. J. Parravicini, D. Pierangeli, F. Di Mei, A. J. Agranat, C. Conti, and E. DelRe, Opt. Express 21, 30573 (2013).

24. J. Toulouse, Ferroelectrics 369, 203 (2008).

25. V. V. Shvartsman and D. C. Lupascu, J. Am. Ceram. Soc. 95, 1 (2012).

26. E. DelRe, B. Crosignani, and P. Di Porto, Prog. Opt. 53, 153 (2009).

27. B. Crosignani, P. Di Porto, A. Degasperis, M. Segev, and S. Trillo, J. Opt. Soc. Am. B 14, 3078 (1997).

28. M. Segev, G. C. Valley, B. Crosignani, P. DiPorto, and A. Yariv, Phys. Rev. Lett. 73, 3211 (1994).

29. E. DelRe, M. Tamburrini, M. Segev, E. Refaeli, and A. J. Agranat, Appl. Phys. Lett. 73, 16 (1998).

30. Z. G. Chen, M. Asaro, O. Ostroverkhova, and W. E. Moerner, Opt. Lett. 28, 2509 (2003).

31. E. DelRe, A. Ciattoni, and A. J. Agranat, Opt. Lett. 26, 908 (2001).

32. E. DelRe, G. De Masi, A. Ciattoni, and E. Palange, Appl. Phys. Lett. 85, 5499 (2004).

33. A. Pierangelo, E. DelRe, E. Palange, A. Ciattoni, Y. Garcia, and A. J. Agranat, Appl. Phys. Lett. 89, 121123 (2006).

34. M. Chauvet, A. Guo, G. Fu, and G. Salamo, J. Appl. Phys. 99, 113107 (2006).

35. D. M. Radheep, D. Mohan, P. Sarkar, S. Arumugam, and P. Mandal, Appl. Phys. Lett. 102, 092406 (2013). 